\title{
First record of Xanthozona melanopyga (Diptera: Tachinidae) on Brassolis astyra (Lepidoptera: Nymphalidae) for Rio Grande do Sul, Brazil
}

\author{
Geraldo Salgado-Neto ${ }^{1,2}$ \\ ${ }^{1}$ Departamento de Fitotecnia, Centro de Ciências Rurais - CCR, Universidade Federal de Santa Maria, \\ Campus Universitário, Camobi, Av. Roraima, $n^{\circ}$ 1000, CEP 97105-900, Santa Maria, RS, Brasil \\ ${ }^{2}$ Corresponding author: Geraldo Salgado-Neto,e-mail: gsalgado@bol.com.br
}

SALGADO-NETO, G. First record of Xanthozona melanopyga (Diptera: Tachinidae) on Brassolis astyra (Lepidoptera: Nymphalidae) for Rio Grande do Sul, Brazil. Biota Neotrop. 11(3): http://www.biotaneotropica. org.br/v11n3/en/abstract?short-communication+bn01711032011

\begin{abstract}
Xanthozona melanopyga (Wiedmann, 1830) (Diptera: Tachinidae) is recorded here as a parasitoid of Brassolis astyra Godart, 1824 (Lepidoptera: Nymphalidae). Dipteran endoparasitoids have been recorded as pupal endoparasitoids of Brassolis sophorae (L.), due to the scientific and economic importance of these interespecific relationships, and aiming to increase the knowledge on natural and conservative biological control, I hereby record Xanthozona melanopyga (Wiedmann) as larval-pupal parasitoid of Brassolis astyra Godart for the State of Rio Grande do Sul, Brazil. For the first time.

Keywords: biological control, distribution, natural enemy, parasitoid flies.
\end{abstract}

SALGADO-NETO, G. Primeiro registro de Xanthozona melanopyga (Diptera: Tachinidae) em Brassolis astyra (Lepidoptera: Nymphalidae) para o Rio Grande do Sul, Brasil. Biota Neotrop. 11(3): http://www. biotaneotropica.org.br/v11n3/pt/abstract?short-communication+bn01711032011

Resumo: Xanthozona melanopyga (Wiedmann, 1830) (Diptera: Tachinidae) é registrada como parasitóide de Brassolis astyra Godart, 1824 (Lepidoptera: Nymphalidae). Representantes de Diptera têm sido registrados como endoparasitóides de pupas de Brassolis sophorae (L.). Diante da importância científica e econômica desse tipo de relação interespecífica, visando à ampliação de conhecimentos relacionados ao controle biológico natural e conservativo, o presente registra no Estado do Rio Grande do Sul, a ocorrência de parasitismo por Xanthozona melanopyga (Wiedmann, 1830) como parasitóide larva-pupa em Brassolis astyra Godart, 1824. Pela primeira vez.

Palavras-chave: controle biológico, distribuição geográfica, inimigo natural, moscas parasitóides. 
Species of Diptera (Tachinidae and Sarcophagidae) (Ferreira \& Lins 2002, Ruszczyk \& Ribeiro 1998) have been recorded as pupal or larvipupal endoparasitoids, acting therefore as biological control agents of Brassolis sophorae (L.), a pest in coconut crops (Ferreira et al. 1998). The present note reporter Xanthozona melanopyga (Wiedmann, 1830) parasitizing pupae of Brassolis astyra Godart, 1824, for the State of Rio Grande do Sul, Brazil.

Brassolis astyra Godart, 1824 is widespread in Brazil but is most commonly found between the States of Rio de Janeiro and São Paulo (Southeastern Region) and Eastern Brazilian Amazonia (Northern Region) (Stichel 1932, Costa Lima 1936, Bondar 1940, Travassos Filho \& Carrera 1941, Silva et al. 1968). In Rio Grande do Sul (Southern Region) this species was registered by Biezanko \& Freitas (1938), Biezanko (1960), Link \& Alvarez Filho (1979), Link et al. (1980) and Lamas (2004). Mariconi (1952) reporter the occurrence of this species for South America (Argentina). More recently, Betancourt \& Scatoni (2002) reported this species for Uruguay.

Five species of Tachinidae have been recorded as parasitoids of Brassolis (pupal or larvipupal stages): Winthemia pinguis (Fabricius, 1805), Winthemia analis (Macquart, 1846), Lespesia haywardi (Blanchard, 1942), Belvosia wiiliamsi (Aldrich, 1928) and Xanthozona melanopyga (Wiedmann, 1830). Previously only Xanthozona melanopyga (Wiedmann) was recorded as endoparasits of species of Brassolis (Cleare \& Squire 1934, Travassos Filho \& Carrera 1941, Piza \& Zamith 1944, Lepesme 1947, Costa Lima 1950, Lordello 1952, Silva et al. 1968, Guimarães 1971, Ruszczyk 1996, Ruszczyk \& Ribeiro 1998, Marcicano et al. 2009).

Between March 2006 and March 2007, we collected "cartuchos" (nest, larvae communal shelter) of Brassolis astyra on native palms Syagrus romanzoffianum (Cham.) Becc. (Arecaceae; Gerivá) in Tupanciretã $\left(53^{\circ} 50^{\prime} \mathrm{W} ; 29^{\circ} 04^{\prime} \mathrm{S}\right)$ and Santa Maria (53 $42^{\prime} \mathrm{W}$; $\left.29^{\circ} 43^{\prime} \mathrm{S}\right)$, Rio Grande do Sul, Brazil.

Upon collecting, larvae were kept in an environmental chamber ( $25 \pm 1{ }^{\circ} \mathrm{C} ; 70 \% \mathrm{UR}$; photoperiod of 14 hours of light) and observed daily until the emergence of the butterflies or parasitoids, which were preserved in $70 \%$ ethanol. Voucher specimens were deposited in the collection of the Laboratorio de Biologia Evolutiva of the Universidade Federal de Santa Maria (UFSM). All parasitoids were identified as Xanthozona melanopyga (Wiedmann, 1830), a species that is known from Central and South America (Brazil, Colombia, Costa Rica and Suriname) (Wiedmann 1830, Townsend 1908, Townsend 1939, Gómez 1998, Bustillo Pardey 2011). In Brazil, the species is known from the States of São Paulo (Travassos Filho \& Carrera 1941, Piza \& Zamith 1944, Costa Lima 1950, Lordello 1952, Mariconi \& Zamith 1954, Silva et al. 1968, Guimarães 1971, Ruszczyk 1996, Ruszczyk \& Ribeiro 1998), Minas Gerais (Clark \& Anjos 1977), Goiás (Mariconi 1971), and Paraná (Santos \& Rocha 1981).

$X$. melanopyga female flies oviposit directly into the larvae during early stage of development, and their larvae subsequently feed on pupal tissues (pupal or larvipupal endoparasitoid). This species is known to be associated to the following butterfly species: Rachiplusia nu (Guenée 1852) (Noctuidae); Hemiceras pallidula (Guenée 1852) (Notodontidae) (Silva et al. 1968) and Brassolinae (Nymphalidae): Caligo illioneus (Gómez 1998, Bustillo Pardey 2011); Opsiphanes invirae (Silva et al. 1968); Brassolis sophorae (Piza \& Zamith 1944, Lepesme 1947, Costa Lima 1950, Lordello 1952, Mariconi \& Zamith 1954, Silva et al. 1968, Guimarães 1971, Mariconi 1971, Clark \& Anjos 1977, Guimarães 1977, Santos \& Rocha 1981, Ruszczyk 1996, Ruszczyk \& Ribeiro 1998).

Although, X. melanopyga has already been recorded as an endoparasitoid of Brassolis astyra in Brazil (State of São Paulo,
Piracicaba, Campinas) (Travassos Filho \& Carrera 1941, Costa Lima 1950, Mariconi \& Zamith 1954, Silva et al. 1968, Guimarães 1971, Guimarães 1977) this constitutes the first record of this species in the State of Rio Grande do Sul.

\section{Acknowledgements}

My thanks to Dr. Alexandre Ruszczyk and Dr. Dionísio Link (CCR/UFSM) for assistance with constant encouragement and friendship.

\section{References}

BETANCOURT, C.M. \& SCATONI, I.B. 2002. Primer register of Uruguay de Brassolis astyra Godart (Lepidoptera, Nymphalidae, Brassolinae). Agrociência 6:87-89.

BIEZANKO, C.M. 1960. Satyridae, Morphidae et Brassolidae da Zona Sueste do Rio Grande do Sul. Arq. entomol., Ser. A (3-4):1-13.

BIEZANKO, C.M. \& FREITAS, R.G. 1938. Catálogo dos Insetos encontrados na cidade de Pelotas e seus arredores. Escola de Agronomia Eliseu Maciel, Pelotas, Boletim 25 - Lepidópteros, fascículo I, 32p.

BONDAR, G. 1940. Insetos nocivos e moléstias do coqueiro (Cocos nucifera) no Brasil. Bol. Inst. Cent. Fomento Ec. Bahia 8:1-60.

BUSTILLO PARDEY, A.E. 2011. Parasitoides, predadores y entomopatógenos que afetan las plagas de la caña de azúcar en Colombia. Cenicana. Ser. tec. 6481:1-11.

COSTA LIMA, A.M. 1936. Terceiro catalogo dos insetos que vivem nas plantas do Brasil. Ministério da Agricultura, Rio de Janeiro, 460p.

COSTA LIMA, A.M. 1950. Insetos do Brasil. Escola Nacional de Agronomia, Rio de Janeiro, Brasil, 6nd tomo. Lepidópteros, parte 2, Série Didática, $420 \mathrm{p}$.

CLARK, E.W. \& ANJOS, N. 1977. A ocorrência, em Minas Gerais, de Xanthozona melanopyga Wied, 1830 (Diptera: Tachinidae), um parasito da lagarta-das-palmeiras Brassolis spp. Lepidoptera: Brassolidae. Viçosa-MG. Rev. Árvore 1(2):176-177.

CLEARE, L.D. \& SQUIRE, F.A. 1934. The coconut caterpillar, Brassolis sophorae L. (Lep., Brassolidae) in British Guyana. Agricult. J. Br. Guyana 5:166-199.

FERREIRA, J.M.S., LIMA, M.F., SANTANA, D.L. Q., MOURA, J.I.L. \& SOUZA, L.A. 1998. Pragas do coqueiro, p.189-267. In A cultura do coqueiro no Brasil (J.M.S. Ferreira, D.R.N. Warwick \& L.A. Siqueira, eds.). 2nd ed. rev. ampl. Embrapa-SPI/Aracaju, Embrapa CPATC, Brasília, 292p.

FERREIRA, J.M.S. \& LINS, P.M.P. 2002. Manejo integrado de pragas. In Frutas do Brasil: coco fitossanidade (E.C. Leal, D.R.N. Warwick, F.B. Sarro, J.M.S. Ferreira, P.M.P. L ins \& R.P.C. Araújo, eds.). Embrapa Informações Tecnológicas, Brasília, p.63-82.

GÓMEZ, L.A. \& LASTRA, L.A. 1998. Caligo illioneus: el gusano cabrito. Cenicana. Ser. tec. 20 (1):8-14.

GUIMARÃES, J.H. 1971. Family Tachinidae (Larvaevoridae). In A Catalogue of the Diptera of the Americas South of the United States (N. Papavero, ed.). Museu de Zoologia, Universidade de São Paulo, São Paulo, v.104, $333 \mathrm{p}$.

GUIMARÃES, J.H. 1977. Host-parasite and parasite-host catalogue of South American Tachinidae (Diptera). Arq. Zool. 28:131.

LAMAS, G. 2004. Atlas of Neotropical Lepidoptera. Association of Tropical Lepidoptera, Scientific Publishers, Gainesville, Checklist: part 4 A, Hesperioidea \& Papilionoidea, 439p.

LEPESME, P. 1947. Les insectes des palmiers. Paul Lechevalier Éd., Paris, $904 p$.

LINK, D. \& ALVAREZ FILHO, A. 1979. Palmeiras atacadas por lagartas de Brassolidae (Lepidoptera) em Santa Maria, RS. Rev. Cent. Cienc. Rurais 9:221-225. 
LINK, D., BIEZANKO, C.M., CARVALHO, S. \& TARRAGÓ, M.F.S. 1980 Lepidoptera de Santa Maria e Arredores. III. Morphidae e Brassolidae. Rev. Cent. Cienc. Rurais 10:191-195.

LORDELLO, L.G.E. 1952. Sobre os inimigos naturais da lagarta das palmáceas Brassolis sophorae sophorae (L.) (Lepidopterae; Brassolidae). An. Esc. Super. Agric. Luiz Queiroz 9:23-30.

MARCICANO, M.D.L., NIHEI, S.S. \& LIMA, I.M.M. 2009. First Host Record for Winthemia analis (Macquart) (Diptera: Tachinidae: Exoristinae) in Brazil: Brassolis sophorae laurentii Stichel (Lepidoptera: Nymphalidae: Brassolinae). Neotrop. Entomol. 38:550-552. http://dx.doi. org/10.1590/S1519-566X2009000400020

MARICONI, F.A.M. 1952. As lagartas das palmeiras. Biológico 18:103-107.

MARICONI, F.A.M. 1971. Inseticidas e seu emprego no combate às pragas. 3nd ed. A Gazeta Maçônica, São Paulo, toma 1, 305p.

MARICONI, F.A.M. \& ZAMITH, A.P.L. 1954. Contribuição para o conhecimento da Brassolis sophorae (Linnaeus, 1758), B. astyra (Godart, 1821) e de seus inimigos naturais. An. Esc. Super. Agric. Luiz Queiroz 212:159-222.

PIZA, S. \& ZAMITH, A. 1944. Contribuição para o conhecimento da organização e da biologia de Brassolis sophorae (Lep. Brassolidae) e de seu parasita Xanthozona melanopyga (Dip. Tachinidae). Rev. Agric. 19(5-6):204-220.

RUSZCZYK, A. 1996. Spatial patterns in pupal mortality in urban palm caterpillars. Oecologia 107:356-363. http://dx.doi.org/10.1007/ BF00328452
RUSZCZYK, A. \& RIBEIRO, J.C. 1998. Mortalidade dos parasitóides Spilochalcis morleyi (Hymenoptera, Chalcididae) e Xanthozona melanopyga (Diptera; Tachinidae) em pupas femininas e masculinas de Brassolis sophorae (Lepidoptera, Nymphalidae). Rev. Bras. Biol. 58:633-637. http://dx.doi.org/10.1590/S0034-71081998000400010

SANTOS, H.R. \& ROCHA, M.A.L. 1981. Controle natural da lagarta das palmeiras (Brassolis sophorae L. 1759), (Lep., Brassolidae, por doenças virulentas e parasitismo de Xanthozona melanopyga (Wied. 1830), Dip., Tachinidae. Universidade Federal do Paraná, Curitiba. Rev. Set. Cienc. Agrar. 3 (2):155-157.

SILVA, A.G.C., GONÇALVES, C., GALVÃO, D.M., GONÇALVES, A.J.L., GOMES, J., SILVA, M.N. \& SIMONI, M.L. 1968. Quarto catálogo dos insetos que vivem nas plantas do Brasil: Seus parasitos e predadores. Ministério da Agricultura, Rio de Janeiro, parte 2, tomo 1, 622p.

STICHEL, H. 1932. Brassolidae. In Lepidopterorum Catalogus (E. Strand). W. Junk, Berlin, v. 51, 115p.

TOWNSEND, C.H.T. 1908. The taxonomy of the muscoidean flies, including description of new genera and species. Smithsonian Miscellaneous Collections, v. 51, 138p.

TOWNSEND, C.H.T. 1939. Manual of Myiology in twelve parts. Townsend \& Filhos, Itaquaquecetuba, part. 7, 434p.

TRAVASSOS FILHO, L. \& CARRERA, M. 1941. Xanthozona melanopyga (Wiedmann, 1830) (Dip. Tachinidae), predadora de Brassolis astyra Godart, 1824 (Lep. Brassolidae), praga das palmeiras. Dados bionômicos dos dois insetos e morfológicos do taquinídeo. Arq. zool. Est. São Paulo 3:43-74.

WIEDMANN, C.R.W. 1830. Aussereuropäische zweiflügelige Insekten. Schulzischen Buchhandlung, Hamm, v. 2, 684p. 